\title{
EL CURRÍCULO NICARAGÜENSE PARA LA ENSEÑANZA DE LA LECTURA EN LA MODALIDAD MULTIGRADO
}

\author{
Nicaraguan Curriculum for Teaching Early Grades Reading \\ in Mixed-classrooms
}

\author{
Melba Castillo-Aramburu ${ }^{1}$ \\ melbacasa@gmail.com
}

\section{Rafael Meza-Duriez ${ }^{1}$}

rafael.meza@doc.uca.edu.ni
${ }^{1}$ Universidad Centroamericana, Nicaragua

Fecha de recepción: 28/02/2020 Fecha de aprobación: 30/03/2020

\section{Resumen}

El estudio analiza el currículo nacional nicaragüense en lo referido a las orientaciones para la enseńanza de la lectura en aulas multigrado y esboza las formas en que el profesorado lo aplica para ofrecer una instrucción efectiva. Se diseñó una investigación cualitativa de tipo exploratorio con dos etapas: a) análisis documental para conocer las orientaciones del currículo sobre la enseńanza de la lectura, en particular la modalidad multigrado; b) estudio de casos para analizar cómo los maestros contextualizan el currículo a esta modalidad. Para este segundo punto, se visitaron seis escuelas multigrado ubicadas en zonas rurales del país. Se entrevistó a sus maestros, y se hicieron observaciones de aula para determinar las influencias del currículo en las prácticas educativas del personal docente. Como resultado, se han identificado importantes áreas de mejora en el currículo nacional en lo que respecta a la enseñanza de la lectura en la modalidad multigrado. Se ha encontrado que el currículo puede limitar las posibilidades de una enseñanza efectiva en estas aulas.

Palabras clave: Educación rural, enseñanza de la lectura, enseñanza primaria, opciones educativas, planificación de la educación.

\section{Abstract}

This study analyses how the national curriculum influences the teaching practices implemented by professors in terms of reading instruction for rural children in multigrade schools. This is a qualitative and exploratory research study carried out in two stages: a) firstly, a documental analysis to understand how the national curriculum addresses reading teaching instruction, particularly for mutigrade settings; b) a case study to evaluate how teachers contextualized the curriculum in schools with this modality. For this second stage, six multigrade schools in rural areas were visited. Teachers were interviewed and classes observed to determine the influences of the national curriculum on the reading instruction process implemented in those schools. In the results, the study identified marginal areas of improvement in the national curriculum in terms of teaching reading in multigrade modality. It was also identified that the curriculum could limit effective instruction in these classrooms.

Keywords: Educational opportunities, educational planning, primary education, reading instruction, rural education. 


\section{Introducción}

La modalidad multigrado es aquella en la cual la enseńanza se desarrolla con alumnos de grados o niveles diferentes que se encuentran en una misma sección y reciben clases al mismo tiempo con un solo maestro (Kivunja, 2014); esto requiere una gestión del aula que alterne estratégicamente la enseñanza directa e indirecta (Ames, 2007). Esta modalidad es utilizada principalmente en zonas rurales de baja densidad poblacional, las que, según Boix (2011), presentan características sociales y culturales que deben ser tomadas en cuenta por la escuela.

La modalidad multigrado es utilizada en muchos países de América Latina, África y Asia; es una opción casi obligada para alumnos de comunidades alejadas de los centros urbanos. Diversas investigaciones realizadas en Vietnam, Uganda (Pridmore, 2007), Zambia (Kivunja, 2014) y la Amazonía peruana (Ames, 2004b, 2004a) mostraron que la uniformidad de currículo y de recursos didácticos, unida a las condiciones de desventaja para el profesorado de multigrado, inciden tanto en la baja calidad de la enseñanza como en la percepción negativa sobre esta modalidad.

En Nicaragua, el $58 \%$ de la matrícula de primaria está localizada en las zonas rurales. A su vez, el 57\% de esa matrícula rural asiste a la modalidad multigrado, lo que representa una población de cerca de 300,000 alumnos, según datos del Ministerio de Educación (MINED). Asimismo, los mayores niveles de pobreza se concentran en las zonas rurales, de ahí la importancia de la matrícula multigrado para estos sectores, ya que constituye la alternativa más accesible.

Según el Centro de Investigación y Acción Educativa Social (CIASES, 2017), Nicaragua carece de un debate informado sobre las políticas educativas, por lo que se desconocen los fundamentos teóricos que promueven reformas de dichas políticas. También ha destacado la falta de acceso a la información pública y la nula recuperación de la memoria histórica, lo que hace difícil entender cómo ha evolucionado pedagógica y metodológicamente la enseñanza de la lectura. Lo que sí se sabe es que el MINED en el año 2015 estableció que el método a utilizar sería el fónico, analítico, sintético (FAS) (MINED, 2019) en todos los centros y modalidades. La razón de esta implementación "en cascada" se basa en que este método ha sido aplicado con éxito en Cuba (MINED, 2015), pero no se sabe mucho más al respecto.

Este estudio se propone, por una parte, analizar las orientaciones del currículo nacional para la enseñanza de la lectura en la modalidad multigrado y, por otra, esbozar cómo está siendo implementado en las aulas de zonas rurales de Nicaragua a las que tuvimos acceso para este estudio.

Para ello se busca responder a la siguiente pregunta: ¿Cómo orienta el currículo nacional la enseñanza de la lectura, para la modalidad multigrado, en zonas rurales y cómo contextualizan los maestros estas orientaciones para enseñar efectivamente en estas aulas?

\section{Revisión de la literatura}

Esta investigación toma como punto de partida el enfoque de lectoescritura inicial (LEI) con que se abordó la revisión sistemática de Kim et al. (2016), y el enfoque llamado Simple View of Reading (SVR) cuyo soporte teórico se encuentra en los fundamentos cognitivos de la lectura (CFRA) (Hoover \& Stone, 2018; Tunmer \& Hoover, 2019).

Este enfoque destaca que el aprendizaje de la lectura está fundamentado en una sólida base de habilidades cognitivas tanto de nivel alto como de nivel bajo. Las habilidades de nivel bajo consisten en el desarrollo de la conciencia fonológica, la asociación fonema-grafema, el concepto de impresión, el conocimiento sintáctico y fonológico, y la decodificación. Por su parte, las habilidades superiores son las siguientes: la realización de inferencias, el relacionamiento de lo leído con los conocimientos previos, la extracción del sentido global y la creación del significado basado en las estructuras previas de conocimiento.

Estas habilidades se organizan según dos grandes componentes necesarios para el dominio de la lectura: el reconocimiento de palabras y la comprensión lectora. 
Según Tunmer y Hoover (2019), ambos componentes contienen habilidades cognitivas subyacentes a los procesos de lectura que tienen una función específica para el dominio de esta competencia. También señalan que, aunque hay jerarquización de estas habilidades cognitivas en ambos componentes, existe complementariedad entre las habilidades cognitivas básicas y superiores, por lo que su enseñanza debe ser integrada.

En la enseñanza de la lectura se conjugan diferentes factores para determinar la efectividad de los procesos de instrucción. Por ello, toda estrategia de enseñanza de lectura debe contemplar el marco de análisis de las cinco T (5T): teaching o enseñanza, tasks o actividades de aprendizaje, tests o evaluaciones, time o tiempo de instrucción y texts o materiales, marco que surge de una adecuación a la propuesta de análisis de Arlington (2002).

Además, existen prácticas cuya eficacia en el aprendizaje de la lectura ha sido comprobada en contextos de pobreza (Guardia, 2018). Se seleccionaron aquellas con un nivel de evidencia entre moderado y fuerte: a) empleo de un modelo balanceado que integre las habilidades de los dos componentes del SVR, b) enseñanza explícita de estrategias de comprensión lectora en la que se emplee modelaje, práctica guiada y práctica autónoma, c) implementación de un sistema fónico como punto de partida para el reconocimiento de palabras, d) evaluaciones de alta calidad para garantizar la progresividad, y e) creación de un clima de lectura motivador.

La complejidad también radica en la necesidad de ajustar el marco teórico de referencia para la enseñanza de la lectura a los procesos sistemáticos para la enseñanza en modalidad multigrado. El modelo de educación sugerido en multigrado parte de que una localidad presenta un conjunto propio de actividades económicas múltiples y condiciones de tipo geográfico, histórico, cultural, paisajístico y ecológico propias, junto con estilos de vida y saberes locales distintos a los urbanos. Estos indicadores forman parte de las señas de identidad de la escuela y pueden (y deben) ser incorporados al currículo escolar (Boix, 2011; Santos Casaña, 2011; Weiss, 2000).
Las evidencias surgidas de investigaciones en contextos rurales de México (Castillo Rojas, 2011; Secretaría de Educación Pública de México, 2005; \& Subsecretaría de Educación Básica [SEB], 2009), Perú (Ames, 2004a, 2007; Montero et al., 2001) y Argentina (Castedo et al., 2012) proponen elementos clave para crear ambientes alfabetizadores en aulas multigrados: a) transversalizar el lenguaje en todas las asignaturas; b) crear rincones de aprendizaje con materiales de lectura variada y adecuada a las edades; c) fomentar la lectura en cada actividad, trascendiendo el aula de clase y la materia específica; y d) incorporar a padres y madres de familia a la vida letrada del estudiantado y al estrechamiento de las relaciones entre comunidad y escuela (Ames, 2004b).

Para incorporar estos procesos, la literatura identifica tres modelos para asegurar la atención directa e indirecta en el aula multigrado (Ames 2006; 2007). Un primer modelo enseńa separadamente a cada grupo, cada uno con sus propios contenidos, niveles y temáticas; Pridmore (2007) lo denomina cuasi-multigrado. En el segundo modelo se enseña de forma conjunta a todos los grupos a partir de una misma temática, es el modelo whole-class (Ames 2006, 2007). El tercer modelo descansa en la alternancia entre el trabajo diferenciado y el trabajo grupal, y se denomina currículo diferenciado (Pridmore, 2007).

\section{Método}

Para responder al tema de investigación se implementó un estudio cualitativo de tipo exploratorio, organizado en dos partes: análisis documental del currículo nacional y estudio exploratorio de seis escuelas multigrado. El estudio exploratorio se elaboró en el marco de una investigación de carácter más amplio realizada por el Centro de Investigación y Acción Educativa (CIASES) con auspicio técnico y financiero del Programa de Capacidades LAC READS, que abarcó 24 escuelas y 38 maestros. De estas escuelas, seis fueron multigrado, con seis maestros y 154 alumnos. De la información obtenida sobre estas escuelas se escribió este artículo que trata sobre esa parte de la investigación. 


\subsection{Análisis documental del currículo nacional}

El análisis documental se centró en la Malla $\mathrm{Cu}$ rricular de Primer Ciclo para Educación Primaria (MINED, 2019) y estuvo orientado por las siguientes preguntas:

- ¿Qué elementos conceptuales propone el currículo sobre la enseńanza de la lectura en la modalidad multigrado?

- ¿Qué actividades propone el currículo para facilitar el aprendizaje de la lectura?

- A partir de sus pautas evaluativas, ¿de qué manera define el currículo una ruta de progresión para el dominio de las habilidades de lectura?

- ¿Cuáles son los textos y materiales que propone el currículo para enseñar a leer a niños y niñas?

- ¿Cuánto tiempo indica el currículo que se le debe dedicar a la lectura?
Para responderlas se construyó una guía de análisis documental, previamente validada en antiguos programas del Ministerio, para entender cómo el currículo nacional incorpora los cinco elementos (en el marco de las 5T) y ver si provee las orientaciones necesarias para enseñar a leer de forma efectiva en las aulas multigrado.

\subsection{Estudio exploratorio de seis escuelas multigrado}

El análisis de las prácticas pedagógicas se realizó en seis escuelas multigrado del área rural, en municipios de difícil acceso, alejados de la capital (Managua), a las que acuden niños de escasos recursos. Estos centros cuentan con una infraestructura deficiente y tienen poco acceso a materiales oficiales. Es importante señalar que la muestra no pudo ser aleatoria debido a las restricciones de acceso que las autoridades gubernamentales han impuesto sobre las escuelas.

Tabla 1. Comparación de escuelas visitadas

\begin{tabular}{|c|c|c|c|}
\hline Código de la escuela & Cantidad de alumnos & $\begin{array}{l}\text { Grados atendidos en el } \\
\text { aula por un solo maestro }\end{array}$ & Materiales observados \\
\hline Escuela 1 & 20 niños y 4 niñas & $\begin{array}{l}\text { Primero a sexto grado (Uni- } \\
\text { docente) }\end{array}$ & $\begin{array}{l}\text { Lápices de grafito, crayones y cuader- } \\
\text { no individual para cada colegial }\end{array}$ \\
\hline Escuela 2 & 22 niños y 10 niñas & $\begin{array}{l}\text { Primero a sexto grado (Uni- } \\
\text { docente) }\end{array}$ & $\begin{array}{l}\text { Lápices de grafito, crayones y cuader- } \\
\text { no individual para cada colegial }\end{array}$ \\
\hline Escuela 3 & 9 niños y 11 niñas & $\begin{array}{l}\text { Primero, segundo y tercer } \\
\text { grado }\end{array}$ & $\begin{array}{l}\text { Lápices de grafito y crayones para } \\
\text { cada colegial, componedor y cuaderno } \\
\text { individuales, libro de texto, cuaderno } \\
\text { de aprestamiento }\end{array}$ \\
\hline Escuela 4 & 15 niños y 11 niñas & Primero y segundo grado & $\begin{array}{l}\text { Lápices de grafito, crayones y cuader- } \\
\text { no individual para cada colegial }\end{array}$ \\
\hline Escuela 5 & 11 niños y 16 niñas & Primero y segundo grado & $\begin{array}{l}\text { Lápices de grafito, y cuaderno indivi- } \\
\text { dual para cada colegial }\end{array}$ \\
\hline Escuela 6 & 12 niños y 13 niñas & $\begin{array}{l}\text { Primero, segundo y cuarto } \\
\text { grado }\end{array}$ & $\begin{array}{l}\text { Lápices de grafito y crayones para } \\
\text { cada colegial, componedor individual y } \\
\text { cuaderno individual }\end{array}$ \\
\hline
\end{tabular}


Los formularios de entrevistas y observaciones de aula fueron validados con maestros de una escuela en Managua, lo que permitió mejorar el instrumento y redactar la versión final. Se hicieron entrevistas a maestros de las escuelas multigrado y firmaron una hoja de consentimiento informado que detallaba el objetivo de la investigación y garantizaba la confidencialidad y anonimato de sus respuestas.

Paralelamente, se realizaron 18 observaciones de aula de las clases de Lengua y Literatura en Letras, en dos momentos del año escolar: a inicios del primer semestre y a inicios del segundo (marzo y agosto). La observación durante la sesión (90 minutos) fue deta- llada en el formulario por uno de los investigadores y un asistente de investigación bajo su supervisión. Se privilegió la observación de las actividades realizadas por cada maestro en primero y segundo grado.

\subsection{Procedimiento de análisis}

Se realizó un análisis de tres niveles. El primer nivel permitió comprender las manifestaciones del fenómeno en cada una de las unidades de análisis, a saber, el currículo, las percepciones e ideario docente, y la práctica de aula. Este primer análisis fue la base para establecer una relación de causa efecto entre las unidades, la misma se explica en la siguiente tabla.

\section{Tabla 2. Etapas del análisis}

\begin{tabular}{llll}
\hline $\begin{array}{l}\text { Análisis individual según } \\
\text { categorías de análisis }\end{array}$ & $\begin{array}{l}\text { Configuración del currículo } \\
\text { Análisis documental }\end{array}$ & $\begin{array}{l}\text { Percepciones e ideario } \\
\text { docentes } \\
\text { Análisis de las entrevistas }\end{array}$ & $\begin{array}{l}\text { Desenvolvimiento en aula de } \\
\text { clases } \\
\text { Análisis de las observacio- } \\
\text { nes de aula }\end{array}$ \\
\hline $\begin{array}{l}\text { Entendimiento de cómo se } \\
\text { enseña a leer en las aulas } \\
\text { multigrado }\end{array}$ & $\begin{array}{l}\text { Percepciones u ideario } \\
\text { docentes ejercen influencia } \\
\text { sobre: }\end{array}$ & $\begin{array}{l}\text { Desenvolvimiento en aulas } \\
\text { de clases }\end{array}$ \\
\hline $\begin{array}{l}\text { Influencia del currículo } \\
\text { en las formas en que se } \\
\text { enseña a leer en las aulas } \\
\text { multigrado }\end{array}$ & $\begin{array}{l}\text { Configuración del currículo } \\
\text { ejerce influencia sobre: }\end{array}$ & Formas en que se enseña a \\
\hline
\end{tabular}

La información de las entrevistas a maestros y las observaciones de aulas fueron recogidas en los formularios provistos para ese efecto y luego, trasladadas a hojas de Excel de forma textual. Las matrices con la información recolectada fueron cuidadosamente elaboradas para visibilizar las correspondencias o el contraste entre cada unidad de análisis (ver tabla 3). 
Revista Caribeña de Investigación Educativa | 2020, 4(2), 7-22

\section{Tabla 3. Categorías de análisis}

\begin{tabular}{|c|c|c|c|}
\hline $\begin{array}{l}\text { Categorías de } \\
\text { análisis }\end{array}$ & Análisis documental & Entrevistas & Observaciones de aula \\
\hline $\begin{array}{l}\text { Enfoque de } \\
\text { enseñanza }\end{array}$ & $\begin{array}{l}\text { 1. Formas de instrucción } \\
\text { 2. Declaración de ambos com- } \\
\text { ponentes para la enseñanza de } \\
\text { la lectura } \\
\text { 3. Integración de contenidos y } \\
\text { materias con la lectura } \\
\text { 4. La ruralidad como objeto de } \\
\text { aprendizaje }\end{array}$ & $\begin{array}{l}\text { 1. Concepciones de lectura y sus } \\
\text { habilidades subyacentes } \\
\text { 2. Concepciones sobre integra- } \\
\text { ción de contenidos para enseñar } \\
\text { a leer } \\
\text { 3. Conocimiento sobre estrate- } \\
\text { gias de instrucción de lectura } \\
\text { 4. Conocimiento sobre formas de } \\
\text { contextualización del currículo }\end{array}$ & $\begin{array}{l}\text { 1. Formas en que se instruye la } \\
\text { lectura } \\
\text { 2. Formas de integración de } \\
\text { contenidos a la lectura } \\
\text { 3. Formas para ajustar el conte- } \\
\text { nido a al estudiantado }\end{array}$ \\
\hline $\begin{array}{l}\text { Actividades de } \\
\text { aprendizaje }\end{array}$ & $\begin{array}{l}\text { 1. Equilibrio entre habilidades de } \\
\text { decodificación y comprensión } \\
\text { 2. Desarrollo de rutinas de pen- } \\
\text { samiento } \\
\text { 3. Circulación de saberes }\end{array}$ & $\begin{array}{l}\text { 1. Conocimientos sobre los } \\
\text { componentes de la lectura y su } \\
\text { enseñanza } \\
\text { 2. Conocimiento sobre estrate- } \\
\text { gias para hacer circular saberes }\end{array}$ & $\begin{array}{l}\text { 1. Diversidad de actividades } \\
\text { 2. Estrategias de enseñanza } \\
\text { directa e indirecta } \\
\text { 3. Uso del entorno como objeto } \\
\text { de aprendizaje }\end{array}$ \\
\hline Evaluaciones & $\begin{array}{l}\text { 1. Tipos de evaluación que pre- } \\
\text { dominan en el currículo } \\
\text { 2. Ruta de progresión definida }\end{array}$ & $\begin{array}{l}\text { 1. Conocimientos sobre los tipos } \\
\text { de evaluación para la lectura } \\
\text { 2. Especialización de las evalua- } \\
\text { ciones }\end{array}$ & $\begin{array}{l}\text { 1. Tipos de evaluación realizada } \\
\text { en el aula } \\
\text { 2. Uso de herramientas de } \\
\text { evaluación como rúbricas o listas } \\
\text { de cotejo }\end{array}$ \\
\hline $\begin{array}{l}\text { Materiales y } \\
\text { recursos }\end{array}$ & $\begin{array}{l}\text { 1. Criterios para seleccionar } \\
\text { materiales } \\
\text { 2. Materiales definidos } \\
\text { 3. Empleo de recursos propios } \\
\text { de la localidad }\end{array}$ & $\begin{array}{l}\text { 1. Criterios para seleccionar } \\
\text { materiales } \\
\text { 2. Concepciones sobre empleo } \\
\text { de los recursos del entorno }\end{array}$ & $\begin{array}{l}\text { 1. Creación de rincones de } \\
\text { aprendizajes } \\
\text { 2. Utilización de los recursos de } \\
\text { la localidad }\end{array}$ \\
\hline Tiempo & $\begin{array}{l}\text { 1. Tiempo que se destina a la } \\
\text { lectura } \\
\text { 2. Formas para optimizar el } \\
\text { tiempo }\end{array}$ & $\begin{array}{l}\text { 1. 2. Tiempo que dedica a las } \\
\text { lecturas } \\
\text { 3. Concepciones para la optimi- } \\
\text { zación del tiempo }\end{array}$ & $\begin{array}{l}1 \text { Momentos dedicados a la lec- } \\
\text { tura durante la sesión de clases }\end{array}$ \\
\hline
\end{tabular}




\section{Resultados}

\subsection{Análisis curricular}

\subsubsection{Enfoque de enseñanza de la LEI y procesos de instrucción contenidos en el currículo}

Según Guardia (2018), para que la enseñanza de la lectura sea exitosa, se debe instruir de forma balanceada, habilidades de reconocimiento de palabras y habilidades de comprensión del lenguaje oral. Si bien el currículo declara expresamente su alineamiento con el enfoque comunicativo para la enseñanza de la lectura (MINED, 2019) es posible afirmar que varía según la etapa del método FAS que se trabaje.

La Malla Curricular (MINED, 2019) para el primer semestre del año organiza los procesos de enseñanza de la lectura de acuerdo con los requisitos del método FAS, a saber, aprestamiento, que se enfoca en el desarrollo de la lectoescritura emergente (60 horas de clase); adquisición (272 horas de clase), cuyo objetivo es desarrollar la habilidad de asociación fonema-grafema; y afianzamiento (100 horas de clase), que está orientado hacia el desarrollo de la comprensión lectora.

En aprestamiento figuran actividades como la siguiente:

El trabajo de las señales convencionales dependerá del lugar donde esté la escuela, si está ubicada en sector urbano se hace un recordatorio de semáforos, cruces peatonales, uso del andén o zonas de seguridad. En las zonas rurales puede auxiliarse de dibujos o láminas (MINED, 2019, p. 72).

Por su parte, en la etapa de adquisición se desarrolla una secuencia didáctica meticulosa, detallada y similar para todas las letras con las que va a trabajar. Esta secuencia se divide en cinco etapas:

- presentar el grafema y el fonema por separado mediante el uso de recursos visuales;

- reconocer el grafema y el fonema en palabras y oraciones partiendo de unidades básicas del sonido para luego hacer combinaciones más complejas;
- leer palabras y oraciones que contemplen el fonema y el grafema mediante el uso del componedor colectivo e individual;

- presentar el grafema en letra cursiva;

- trazar el grafema en mayúscula y minúscula en el cuaderno de escritura.

Finalmente, en la etapa de afianzamiento se mantiene el carácter vago e impreciso en las formulaciones, ejemplificado en esta actividad de segundo grado: "Lea $[$ sic] comprensivamente cuentos e identifica los personajes y hechos presentes" (MINED, 2019, p. 93). Estas formulaciones imprecisas carecen de suficientes pautas metodológicas a seguir por el maestro.

El currículo no emplea un enfoque balanceado debido al tratamiento metodológico que recibe cada una de esas etapas. Según el modelo SVR (Tunmer \& Hoover, 2009), queda en tela de juicio la idoneidad del currículo para desarrollar la comprensión lectora, ya que se evidencia que plantea estas habilidades de forma lineal y aisladas entre sí, no de forma integrada según lo que propone el modelo. Asimismo, este hallazgo incluso podría aplicarse a los procesos de enseńanza de la modalidad regular.

Salvo para la etapa de adquisición en actividades específicas de asociación fonema-grafema, el maestro no recibe orientaciones para realizar modelaje, práctica guiada ni práctica autónoma (Gutiérrez-Braojos \& Salmerón-Pérez, 2012) de la instrucción directa de las otras habilidades fundamentales implicadas en el aprendizaje de la lectura. Esto contradice también la evidencia internacional (Guardia, 2018) sobre la importancia de desarrollar instrucción directa para la enseńanza de la lectura, especialmente durante los procesos de enseńanza de estrategias de comprensión lectora.

Por otra parte, se observa una confusión conceptual en el currículo entre actividades de aprendizaje y actividades de evaluación. En segundo grado, hay una actividad de aprendizaje que señala: "Lea [sic] diferentes textos e identifica palabras con las sílabas complejas bl, br y sílabas mixtas terminadas en 
d" (MINED, 2019, p. 89). Luego, en actividades de evaluación: "Participa en la lectura de diferentes textos e identifica palabras con las sílabas complejas bl, br y sílabas complejas mixtas terminadas en d" (MINED, 2019, p. 90). Se puede apreciar que las actividades de aprendizaje son más bien de tipo evaluativo pero están enfocadas en la memorización y carecen de aprendizaje significativo, una práctica poco efectiva (Arlington, 2002) por cuanto no garantiza una ruta de progresión clara en el dominio de las habilidades de lectura ni plantea tampoco el desarrollo de procesos de instrucción directa, práctica guiada práctica autónoma (Gutiérrez-Braojos \& Salmerón-Pérez, 2012).

Los materiales más recurrentes son componedor individual y colectivo (lámina o cuadro para que los alumnos vayan colocando las letras), libro de texto, cuaderno de aprestamiento y cuaderno de escritura pautado, materiales propios del método FAS. Falta en el currículo la posibilidad de emplear textos o materiales significativos, esto es, textos, narraciones o leyendas vinculados a las prácticas de cultura escrita de la localidad.

Según Arlington (2002), las aulas efectivas son aquellas en las que el profesorado no se limita al manual o libro de texto. Al contrario, la efectividad de la enseńanza se da en ambientes en los que los alumnos tienen diversas opciones de lectura, ajustadas a su nivel. No es posible saber si los materiales propuestos por el currículo corresponden con los criterios de diversidad y adecuación al nivel del estudiantado de las aulas multigrado.

\subsubsection{Transversalización de la ruralidad en el cu- rrículo}

En el currículo no se evidencian orientaciones específicas destinadas al profesorado para integrar la enseńanza de la lectura con otras materias, puesto que son los contenidos los que determinan los procesos de enseñanza. Pero estos contenidos se basan, ya sea en la letra o grupo fonemático que toca estudiar en un período específico, o en el tipo de texto que se pretende que el estudiantado aprenda.
No se recuperan temas propios del entorno rural, ni sus particularidades sociales y culturales (Boix, 2011), lo que demuestra que existe un distanciamiento entre la cultura escrita que se pretende implementar en la escuela y aquella del entorno de procedencia de los alumnos. Esto contradice lo que indica Ames (2004a) sobre la importancia de articular contenidos con el mundo y las prácticas de cultura escrita de niños y niñas rurales.

Por ende, es posible plantear que los requisitos del método FAS en el currículo limitan la circulación de saberes (Santos-Casaña, 2011), circulación que se da mediante la implementación de actividades comunes o complementarias entre los grados. Cuando los alumnos de primer grado concluyen las seis semanas de aprestamiento y comienzan la adquisición, segundo grado ya ha comenzado los primeros contactos con cuentos y otros tipos de textos. Además, no hay en el currículo ninguna orientación para realizar actividades de interaprendizaje entre los grados.

Pridmore (2007) y Taole (2017) reconocen que, para facilitar la adaptación curricular, el maestro debe ser capaz de integrar contenidos y de alternar actividades diferenciadas para garantizar que, aunque los temas sean afines o comunes, los niveles de desarrollo de habilidades estén diferenciados y ajustados al nivel de cada grupo y cada estudiante. Según el marco de referencia utilizado, las estrategias deben traducirse en actividades de lectura diferenciadas y alternadas que faciliten el aprendizaje colaborativo y la circulación de saberes. Sin embargo, al estar configuradas de acuerdo con las etapas del método FAS, no parece viable que el estudiantado de primero pueda encontrar temáticas y actividades afines y/o complementarias con el segundo grado. Dicho de otro modo, no existen en el currículo posibilidades de alternar estrategias de enseñanza directa e indirecta (Ames, 2006; 2007).

Respecto a la creación de ambientes letrados, no se observó que el currículo orientara la creación y utilización óptima de rincones de aprendizaje, ni formas de transversalizar elementos vinculados a la expresión oral y escrita con el fin de crear ambientes 
letrados que fomenten permanentemente la lectura (SEB, 2009). La etapa de aprestamiento conlleva actividades de esfuerzo conjunto para crear ambientes motivadores para el estudiantado de primer grado. No obstante, su objetivo está más orientado a la adaptación a la cultura escolar que al fomento de la lectura.

Desde nuestra perspectiva, la configuración del currículo, cuya base fundamental es implementar el método FAS, no tiene la intención de recuperar la ruralidad como eje transversal.

El trabajo de las familias es mencionado en la etapa de aprestamiento para asegurarle al estudiante un buen recibimiento en la escuela, pero omite su apoyo en las actividades específicas de lectura. Tampoco hay en el currículo actividades que sugieran el desarrollo de reuniones o encuentros estudiantiles sobre temas libres para potenciar interés y curiosidad por el contexto y la localidad (Secretaría de Educación Pública, 2005).

Consecuentemente, se observa que el currículo propicia el modelo de adaptación cuasi-multigrado (Pridmore, 2007), que consiste en el agrupamiento diferenciado de alumnos según los grados, y cuya implementación tiende a provocar disparidad en la distribución del tiempo entre los grados que se deben atender (Ames, 2004a). En palabras sencillas, los niños y niñas rurales deben estudiar exactamente lo mismo que sus pares de escuelas urbanas regulares, solo que con un maestro que debe limitar el tiempo de instrucción por tener varios niveles a los cuales instruir. Lo anterior es evidencia de que la implementación del método FAS nunca tomó en consideración las particularidades de la enseñanza en aulas multigrado.

\subsection{Prácticas docentes de adaptación curricular encontradas en las entrevistas y observaciones}

\subsubsection{Enfoque de enseñanza}

Es posible identificar en el ideario de docentes y en las prácticas de aula una confusión conceptual alrededor de la lectura como competencia a desarrollar. En general, los maestros reducen el concepto de lec- tura a la decodificación, lo que se evidencia en algunas entrevistas sobre las habilidades necesarias para el desarrollo de la lectura:

[Para desarrollar la lectura se necesita] saber analizar la lectura, desde interpretar lo que están leyendo hasta con ejemplo de los dibujos, trabajamos con las vocales para que digan que vocal está acorde al dibujo que están viendo (JT, comunicación personal, 15 de marzo de 2019).

En los registros se evidencia el énfasis puesto en la decodificación en detrimento de la comprensión, expresando una correlación entre el currículo y las prácticas. Hoover y Stone (2018) y Tunmer y Hoover (2019) destacan que la falta de desarrollo de uno de los dos componentes implica un aprendizaje incompleto de las habilidades de lectura. Más aún, Tunmer y Hoover (2019) enfatizan en que los vacíos en el aprendizaje de habilidades de comprensión del lenguaje y reconocimiento de palabras tienden a potenciar el efecto Matthew en su sentido negativo, esto es, el rezago constante de habilidades de lectura a lo largo de la vida. Véase el ejemplo a continuación:

La clase [...] se enfoca en los sonidos de las vocales y a partir de láminas y palabras. Emplea las manos para que los nińos midan la extensión de la palabra. Se usan aplausos también para identificar sílabas. En segundo grado, los niños transcriben un cuento. Leen de forma independiente y en silencio mientras copian, y luego responden a preguntas planteadas por la docente, las que son de tipo literal (Observación $n^{\circ} 1$ A, marzo de 2019).

Aunado a ello, los maestros parecen ignorar las posibilidades que brinda el entorno para integrar contenidos y transversalizar la ruralidad y convertirla en objeto de aprendizaje (Montero et al., 2001). Al analizar las entrevistas, se identificó el rol limitado del entorno:

Por lo menos con los padres, que saben ayudarles en lo que es la escritura y también en la lectura, porque si nosotros que estamos aquí 
en la escuela practicamos lo que es la escritura y la lectura y en la casa no, entonces, no habría ningún avance (ACT, comunicación personal, 13 de marzo de 2019).

Sí, y ahí aprovecho, porque el jueves vamos hacer los componedores y van a venir ellos, todos los de primer grado. Y también a veces viene un padre de familia, que se llama Andrés y él se mete adentro y dice voy a ir ayudarle a ese chavalo, y se mete y me ayuda (EPO, comunicación personal, 12 de marzo de 2019).

Es evidente que hace falta incorporar con mayor sistematicidad el trabajo con la comunidad (Montero et al., 2001). En ninguna de las observaciones se pudo apreciar la participación de las familias o de otras personas de la comunidad. Esto podría explicarse a partir de las concepciones que tiene el profesorado respecto a lo que pueden aportar las familias, dados los bajos niveles de escolaridad. En todo caso, no se aprecia una intencionalidad al incorporar a las familias, sino que se da de forma aislada y espontánea.

Desde esta concepción, los maestros desaprovechan la posibilidad de solicitar a las familias que compartan experiencias para fortalecer las habilidades lectoras de sus alumnos. Algunos maestros reconocen que invitan a las familias a las sesiones de clases con el ánimo de supervisar y controlar el progreso del estudiante. También destacan que la incorporación de las familias se refleja en el apoyo y el esfuerzo para enviarlos a clases, y en la participación para elaborar los componedores.

\subsubsection{Actividades de aprendizaje, evaluaciones y tiempos de lectura optimizados}

Los maestros entrevistados manifestaron que priorizan el acompańamiento pedagógico en primer grado en detrimento de los otros grados bajo su cargo. Esto se evidencia en dos dinámicas que confirman esta apreciación.

En la primera, se orientan actividades independientes en segundo y tercer grado, para luego trabajar con primer grado la mayor parte del tiempo, como se puede ver en el ejemplo de un registro de observación en una escuela:

Comienza la clase organizando el grupo de segundo grado, mientras los niños de primero esperan a que termine sin hacer ninguna actividad relacionada con lectura. Cuando termina de orientar el trabajo en segundo grado, separa a dos niños de ese nivel que aparentemente tienen rezagos en cuanto a aprendizaje y les orienta un trabajo de trascripción de oraciones. Después de 7 minutos, empieza a trabajar con los de primer grado, a quienes prioriza a lo largo de la hora de clase (Observación $\mathrm{n}^{\circ} 7 \mathrm{~A}$, marzo de 2019).

La segunda dinámica es inversa a la anterior. Véase el siguiente registro de una observación en la escuela de León:

La docente inicia con primer grado. De 8 a $8: 20$. En ese tiempo, los niños de segundo grado esperan a que la profesora les dé indicaciones (no hacen nada). Les explica a los de segundo en 4 minutos su tarea, que consiste en ordenar el nombre de los nińos de primer grado según el alfabeto, y luego regresa a trabajar con primero. Vuelve a acercarse a segundo grado a las 8:43 para supervisar el trabajo que han realizado, mientras primer grado se queda trabajando unas hojas de ejercicio en su cuaderno. En total, fueron 60 minutos de clases, de los cuales, primer grado tuvo 43 minutos de conducción directa, y segundo grado, 17 minutos (Observación nº 8A, marzo, 2019).

Al igual que con el currículo, en las aulas de clases predomina un modelo de adaptación cuasi-multigrado (Pridmore, 2007). Ames (2004a, 2006) destaca la importancia de que los maestros puedan promover el autoaprendizaje y el interaprendizaje. Además, Pridmore (2007) y más recientemente Taole (2017) reconocen que los maestros deben poder mapear el currículo en busca de contenido afines o comunes, así como establecer dinámicas de aprendizaje en las que se pueda trabajar entre todos los grupos que conforman la sección, trabajar cada grupo por su cuenta, o inclu- 
so trabajar individualmente. No obstante, es evidente que la configuración curricular incide en el desarrollo de estrategias de conducción directa e indirecta.

El hecho de que en las aulas de clases predomine un modelo de adaptación curricular cuasi-multigrado incide en la priorización del profesorado en primer grado y limita el factor tiempo (Arlington, 2002), no solo en la instrucción de los alumnos de grados superiores, sino en la creación de espacios dedicados a la lectura silenciosa, coral, entre pares o en voz alta. Las actividades priorizan la conducción directa con primer grado, mientras que las indirectas se aplican principalmente con los grados superiores. Esto limita la circulación de saberes (Santos-Casaña, 2011), que es una condición imprescindible para enseñar efectivamente en esta modalidad.

Lo anterior provoca que las actividades de aprendizaje sean actos de mero trámite, sin continuidad en el tiempo y sin posibilidades de generar desafíos cognitivos (Arlington, 2002) a alumnos, sobre todo de grados superiores a primero. Por extensión, la ruta de progresión del dominio de la lectura se detiene una vez finalizado primer grado.

Ello supone la frecuencia de "tiempos muertos", un claro indicador de una deficiente administración del tiempo. Esto es algo que los maestros reconocen y, en ocasiones, es una realidad imposible de erradicar completamente.

Algunas veces por la falta de recursos, porque si estuviera en nuestro alcance tener siempre una lámina, algo para pintar, algún material en el aula evitáramos esos tiempos muertos (JPBT, comunicación personal, 20 de marzo de 2019).

Los maestros manifestaron que para superar momentos de "tiempo muerto" se apoyan en alumnos más avanzados que se encargan de dirigir al grado a partir del cuaderno de planificación o de guías de aprendizaje.

En cada grado tengo dos monitores, esos monitores tienen las guías que yo hago diario para cada grado por área, ellos les dictan a los otros las actividades y a los que tienen problemas para resolver, ellos le ayudan, para que a mí me dé más tiempo en primer grado, casi sirven como maestros, son los segundos míos, porque ellos son los que chequean al que va avanzando (EPO, comunicación personal, 12 de marzo de 2019).

Bueno, mire los monitores son los niños más avanzados en esa área, [...], a mí se me facilita en ese aspecto porque les oriento el trabajo, quedan trabajando solos, después queda el monitor, voy le reviso y con el apoyo de él, le reviso a los demás. Y después me cercioro si el niño revisó correctamente (JT, comunicación personal, 15 de marzo de 2019).

Además, los maestros no tienen tiempos definidos para la lectura en sus diferentes modalidades (coral, grupal, mediante tutoría o individual). Al preguntarles sobre este tema, los maestros dicen dedicarle entre 45 y 90 minutos a la semana, lo que coincide con las horas clase prescritas en el currículo para la asignatura de Lengua y Literatura, y no necesariamente implica que esas horas clase serán destinadas a la lectura. Ni el currículo ni el profesorado definen tiempos específicos para la lectura. Este tiempo entonces queda a criterio del maestro de turno y de sus hábitos de lectura. El problema con esta configuración es que, según las entrevistas, los maestros atribuyen a la lectura un valor instrumental.

Porque por medio de la lectura yo voy descubriendo algo nuevo que tengo que enseńar, por medio de esa lectura, si yo estoy leyendo un libro como que me llama la atención, aquí hay cómo redactar oraciones, cómo voy a redactar esta oración, entonces digo yo, aquí hay algo bonito para los chavalos (CA, comunicación personal, 19 de marzo de 2019).

Por ejemplo, si yo les voy a traer un cuento a mis niños, yo lo tengo que leer primero para saber el contenido y qué preguntas orales voy a hacer yo, y escritas también; no puedo venir aquí inventando y decirles a los niños van a leer y es cualquier cosa, lo primero que me sa- 
lió; entonces, hasta las lecturas se planean, qué lecturas vamos a trabajar, qué texto (JT, comunicación personal, 15 de marzo de 2019).

Según Arlington (2002), la enseñanza efectiva de la lectura implica disponer más del $50 \%$ del tiempo de clases para leer. Pero no solo para leer el manual de texto o para fines de algún trabajo, sino para lectura de ocio y placer. En este caso, ninguna de las aulas observadas dispuso de tiempo de lectura por placer, lo que contradice la evidencia internacional.

\subsubsection{Materiales y ambientes letrados}

$\mathrm{Al}$ analizar los registros de observación, no se identificaron actividades que promuevan el lenguaje oral tales como diálogos, exposiciones, lectura grupal e individual, o trabajos de escritura con significado, esta carencia limita las posibilidades de crear ambientes letrados (SEB, 2009) y se debe al enfoque que predomina para enseñar a leer.

Según Ames (2004a), para favorecer estos ambientes es necesario crear rincones de aprendizaje que verdaderamente sean de utilidad para el estudiantado. En el caso que nos corresponde, cada escuela dice contar con un centro de recursos de aprendizaje en el aula, pero las de mayor escasez de recursos se limitan a crearlos a partir del abecedario y el pautado para la escritura. Esto obliga a los maestros a hacer un mayor esfuerzo para conseguir el material. Esto se puede ver en la siguiente entrevista:

[El rincón de aprendizaje de lenguaje] es el que está aquí, no tiene mucho, solo los cuentos, hice una lista de cuarenta cuentos, los emplastiqué y son los que estamos ocupando para la comprensión lectora más que todo en segundo y tercero, las letras ahí están cerquita del Centro de Recursos de Aprendizaje, las letras también están, el abecedario (YMM, comunicación personal, 11 de marzo).

No obstante, sí fue posible identificar que los maestros de multigrado realizan un gran esfuerzo para compensar la falta de materiales escritos propios de estas zonas.
Bueno, yo siempre le digo a ellos que, si no tienen un libro a mano, en todas las casas yo sé que tienen biblias, porque en las entrevistas que hemos hecho siempre pregunto ¿Y van a la iglesia? Sí; ¿Y tienen Biblia?, Sí, entonces nadie puede decir que no tiene un libro (JT, comunicación personal, 15 de marzo de 2019).

Este esfuerzo, sin embargo, debe corresponderse con un trabajo más sistemático en el aula de clases para incorporar recursos propios de la cultura escrita en la que se desenvuelve el estudiantado (Ames, 2003a, 2007). En el siguiente registro se aprecian los materiales que predominan en la enseńanza de la lectura en las aulas multigrado.

La docente en primer grado emplea láminas con imágenes que inician distintos grupos vocálicos. Una vez que han terminado el análisis fonético, emplean el cuaderno de escritura para practicar trazos. En caso de segundo grado, la docente utiliza el manual para leer en voz alta y que los niños tengan una idea de cómo se realiza una descripción (Observación 6A, marzo de 2019).

\section{Discusión y conclusiones}

Se ha evidenciado que el currículo nacional no se ajusta a las recomendaciones que dicta la evidencia, tanto para la enseńanza de la lectura (Arlington, 2002; Guardia, 2018; Hoover \& Stone, 2018; Kim et al., 2016; Tunmer \& Hoover, 2019), como para el desarrollo de procesos sistemáticos efectivos en la modalidad multigrado (Ames, 2004a, 2006; Montero et al., 2001; Pridmore, 2007; Taole, 2017).

Pridmore (2007) explica que en los países en desarrollo se emplea casi de forma universal el modelo cuasi-multigrado, lo que coincide con los hallazgos del caso de Nicaragua. Ames (2006) identificó que la modalidad cuasi-multigrado - por el agrupamiento diferenciado de alumnos según los grados - limita la capacidad de atender directamente a los alumnos, o bien provoca una disparidad en la distribución del tiempo entre los grados a atender. Esto parece ser el caso de Nicaragua, ya que se pudo apreciar que los 
alumnos de los grados superiores consumen su tiempo en actividades mecánicas como copiar sílabas o palabras.

Mediante las observaciones y las entrevistas se identifica que en las aulas multigrado se desarrolla una metodología similar a la que identificó Ames (2006) en Perú. Dada esta correspondencia, y en consonancia con lo dispuesto en el currículo, es posible considerar que el método FAS presenta limitaciones para las necesidades didácticas de la modalidad multigrado.

El método FAS, insignia del currículo nacional, no fue diseńado para la modalidad multigrado, por cuanto no contempla procesos de conducción indirecta y su énfasis está puesto en primer grado. La configuración de las aulas multigrado, en las que pueden llegar a coexistir alumnos de seis niveles diferentes a la vez, dificulta que el maestro atienda los procesos de aprendizaje del resto de niveles si se enfoca en primer grado.

En cuanto a instrucción para la enseñanza de la lectura, en el currículo se evidencia un desequilibro de los componentes que subyacen a la comprensión lectora, ya que prioriza las habilidades de decodificación. Esto coincide con los hallazgos realizados por CIASES (2017) y Hoover y Stone (2018) y se traduce también en las prácticas de aula en las que los procesos de comprensión son casi inexistentes.

Otra desventaja es el distanciamiento de la cultura escolar con la de procedencia del estudiantado, distanciamiento que se evidencia en la poca o nula participación de las familias así como en el uso exclusivo de los materiales que dispone el currículo, propios del método FAS. "Rara vez pensamos en personas, y menos aún en los propios niños/as y en sus comunidades, en los saberes que ellos tienen y que pueden enriquecer nuestra labor educativa” (Ames, 2007, p. 31).

La transversalización de la ruralidad requiere la amplia participación de la comunidad en el aprendizaje de los alumnos. La incorporación de padres y madres de familia a la vida letrada del estudiantado incide considerablemente en el desarrollo de las habilidades lectoras y de comprensión, por lo que se busca estrechar la relación entre escuela y comunidad para poder utilizar la realidad local como instrumento y contenido educativo. No obstante, de las entrevistas se intuye que las percepciones docentes parecen estar condicionadas por los bajos niveles de escolaridad de la región, tal como señala CIASES (2017).

En consecuencia, es posible afirmar que la uniformidad del currículo en contenidos, metodologías y materiales incide negativamente en la enseñanza de la lectura en aulas multigrado. Estamos frente a un currículo pensado desde (y para) contextos urbanos y escuelas regulares. La implementación de un currículo único dificulta considerablemente las posibilidades de adaptación y supone para los maestros una carga adicional de trabajo para contextualizarlo a su realidad.

De mantenerse este modelo, se corre el riesgo que persista en el ideario de los nicaragüenses la concepción errónea de considerar a la escuela multigrado como una educación de segunda categoría o carente de calidad.

No obstante, es importante reconocer el esfuerzo que los maestros realizan para adaptar contenidos y orientaciones curriculares para esta modalidad. Para corresponder a esos esfuerzos, es necesario revisar los modelos de formación profesional para brindarles más y mejores herramientas para adaptar los contenidos y disminuir los efectos provocados por la implementación del currículo único.

A partir de estos hallazgos, se considera necesario:

- Comprobar con una muestra de mayor tamaño y ampliada a otras regiones del país, la evaluación de idoneidad del método FAS para enseńar efectivamente en aulas multigrado, y verificar si su aplicación se corresponde con lo esbozado en esta investigación exploratoria.

- Fomentar procesos de formación que surjan desde las necesidades propias de la ruralidad y de los requisitos de la modalidad multigrado, lo que implica destinar recursos para la preparación de docentes especializados en esta modalidad. 
- Promover una reforma curricular a través de procesos de consulta amplios, incluyentes y representativos para la construcción de un currículo flexible que facilite la adaptación de sus orientaciones al territorio rural y a la modalidad multigrado.

- Señalar la necesidad de que el currículo contenga orientaciones sobre actividades dirigidas a fortalecer la enseñanza de la conciencia fonológica e, igualmente, ofrecer estrategias más efectivas para la enseńanza de la comprensión lectora.

- Facilitar a los maestros las estrategias de conducción indirecta que apoyen la trayectoria de progreso de los alumnos más avanzados. Por ejemplo, videos con modelajes sobre estrategias de comprensión lectora $\mathrm{u}$ otras habilidades.

- Asegurar a los maestros los recursos pedagógicos necesarios para una enseńanza efectiva de la lectura e, igualmente, favorecer procesos de capacitación efectivos que les permitan un mejor dominio del método y sus adaptaciones.

- Proponer a los maestros la dedicación de un tiempo determinado (de 20 a 30 minutos) exclusivamente para la lectura.

\section{Agradecimientos}

Expresamos nuestro agradecimiento a la Red para la Lectoescritura Inicial de Centroamérica y el Caribe (REDLEI) por el apoyo financiero y metodológico para la realización de este estudio. Igualmente, queremos agradecer al CIASES y al Programa de Capacidades LAC READS, que nos permitió utilizar la información obtenida de las entrevistas y observaciones de maestros de escuelas multigrado. Igualmente, y de manera especial, a las organizaciones, directores y maestros que participaron activamente en la investigación.

\section{Referencias bibliográficas}

Ames, P. (2004a). Las escuelas multigrado en el contexto educativo actual: desafios y posibilidades. Lima: GTZ-PROEDUCA-Componente de Educación Bilingüe Intercultural.

Ames, P. (2004b). Multigrade Schools in context: literacy in the community, the home and the school in the Peruvian Amazon [Tesis doctoral, University of London]. UCL Discovery.

http://r.issu.edu.do/l.php?1=47t2v

Ames, P. (2006). A multigrade approach to literacy in the Amazon, Peru: School and Community Perspectives. En A. W. Little (Ed.), Education for All and Multigrade Teaching: Challenges and Opportunities (pp. 43-66). Dordrecht: Springer.

Ames, P. (2007). Escuelas Multigrado. Una alternativa para niños y niñas que transitan de preescolar a primer grado. Serie: Educación para la transición con excelencia. Managua: USAID/Save The Children.

Arlington, R. L. (2002). What I've Learned about Effective Reading Instruction. Phi Delta Kappan, 83(10), 740-747.

https://doi.org/10.1177/003172170208301007

Boix, R. (2011). ¿Qué queda de la escuela rural? Algunas reflexiones sobre la realidad pedagógica del aula multigrado. Profesorado, Revista de Curriculum y Formación del Profesorado, 15(2), 13-23.

http://r.issu.edu.do/l.php?l=45qR8

Castedo, M., Peláez, A., Dapino, M., \& Hoz, G. (2012, 5-7 de septiembre). Construcción e intercambio en situaciones de lectura y escritura en aulas multigrado rural. I Congreso Iberoamericano de las Lenguas en la Educación y en la Cultura, Salamanca, España. http://r.issu.edu.do/l.php?l=48aa6

Castillo, M., Castro, V., Elvir, A. P., Vijil, J., \& Lacayo, M. R. (2008). La Educación Rural Nicaragüense: Hacia un diagnóstico de Sus desafios y posibilidades. Managua: CIASES.

http://r.issu.edu.do/l.php?l=253H1 
Castillo-Aramburu, M., Elvir-Maldonado, A. P., \& Vijil-Gurdián, J. (2016). Prioridades de la educación nicaragüense en el siglo XXI. Managua: CIASES. http://r.issu.edu.do/l.php?l=265rP

Castillo-Rojas, A. Y. (2011). Manual para favorecer el desarrollo de competencias de lectura y escritura. Primer ciclo. México D. F.: Secretaría de Educación Pública.

Centro de Investigación y Acción Educativa Social (CIASES). (2017). Programa de Capacidades LAC READS: perfil de pais Nicaragua y análisis de actores clave en lectoescritura inicial. Managua: American Institutes for Research (AIR).

Grigsby, C. (2012). La educación rural en Nicaragua: una aproximación a la realidad de la escuela multigrado en León, Jinotega, Boaco y la RAAS. Managua: IEEPP.

Guardia, P. (2018). Evidencias 41: ¿Qué funciona para reducir las brechas socioeconómicas tempranas en lectura? Evidencia desde revisiones sistemáticas y plataformas What Works. Chile: Ministerio de Educación del Gobierno.

Gutiérrez-Braojos, C., \& Salmerón-Pérez, H. (2012). Estrategias de comprensión lectora: enseñanza y evaluación en educación primaria. Profesorado: Revista de curriculum y formación del profesorado, 16(1), 184202. http://r.issu.edu.do/l.php?l=41b1B

Hoover, W., \& Stone, R. (2018). Marco de bases cognitivas para la lectura. [Manuscrito no publicado].

Instituto de Estudios Estratégicos y Políticas Públicas (IEEPP). (2017). Calidad educativa en la escuela multigrado: Análisis de equidad en el contexto rural. Managua: IEEPP.

Kim, Y. S. G., Boyle, H. N., Zuilkowski, S. S., \& Nakamura, P. (2016). Landscape Report on Early Grade Literacy. Washington, D. C.: USAID.

Kivunja, C. (2014). The urgent need to train teachers for multigrade pedagogy in african schooling contexts: Lessons from Uganda and Zambia. International Journal of Higher Education, 3(2), 63-72. https://doi.org/10.5430/ijhe.v3n2p63
Ministerio de Educación de Nicaragua (MINED). (2015). Método Fónico Analitico Sintético. Orientaciones metodológicas. Managua: MINED.

Ministerio de Educación de Nicaragua (MINED). (2019). Mallas Curriculares de Primer Ciclo para Educación Primaria. Managua: MINED.

Montero, C., Oliart, P., Ames, P., Cabrera, Z., \& Uccelli, F. (2001). La escuela rural: Modalidades y prioridades de intervención. Lima: Programa MECEP.

Popota-Ochoa, C., Hernández-Saucedo, M., \& Reyes-Hernández, M. de L. (2003). La lectura en aula multigrado. México D. F.: Secretaría de Educación Pública.

Pridmore, P. (2007). Adapting the primary school curriculum for multigrade classes in developing countries: a five-step plan and an agenda for change. Journal of curriculum studies, 39(5), 559-576. https://doi.org/10.1080/00220270701488093

Santos-Casaña, L. (2011). Aulas multigrado y circulación de los saberes: especificidades didácticas de la escuela rural. Profesorado, Revista de currículum y formación del profesorado, 15(2), 71-99.

http://r.issu.edu.do/l.php?l=42is7

Secretaría de Educación Pública. (2005). Propuesta Educativa Multigrado 2005. México D. F.: Constantine Editores S.A. de C.V.

Shanahan, T. (2005). The National Panel Reading Report: Practical Advice for Teachers. Estados Unidos: Learning Point Associates.

Subsecretaría de Educación Básica (SEB) (2009). Modelo Educativo Multigrado. Documento de trabajo. México: SEB.

Taole, M. J. (2017). Identifying the professional knowledge base for multigrade teaching. The Online Journal of New Horizons in Education, 7(4), 42-51. http://r.issu.edu.do/l.php?l=43dD1

Tunmer, W. E. \& Hoover, W. A. (2019). The cognitive foundations of learning to read: a framework for preventing and remediating reading difficulties, Aus- 
tralian Journal of Learning Difficulties, 24(1), 75-93. https//doi.org/10.1080/19404158.2019.1614081

Weiss, E. (2000). La situación de la enseñanza multigrado en México. Perfiles Educativos. 22(90), 57-76. http://r.issu.edu.do/l.php?l=44gVJ

\section{CÓMO CITAR:}

Castillo-Aramburu, M., \& Meza-Duriez, R. (2020). El currículo nicaragüense para la enseñanza de la lectura en la modalidad multigrado. Revistas Caribeña de Investigación Educativa (RECIE), 4(2), 7-22. https://doi.org/10.32541/recie.2020.v4i2.pp7-22 